\title{
Magnetic characterization of (001) and (111) Ni films epitaxially grown on MgO
}

R. A. Lukaszew ${ }^{1}$, V. Stoica and R. Clarke Randall Laboratory of Physics, University of Michigan, Ann Arbor, MI 48109

${ }^{1}$ Department of Physics and Astronomy, University of Toledo, Toledo, OH 43606

\begin{abstract}
One interesting application of epitaxial magnetic thin films is to use them as one of the electrodes in a spin-dependent tunneling junction, in order to use the magnetocrystalline anisotropy to define the required two states of the magnetization. [1] In our preliminary work, we prepared epitaxial magnetic films on copper buffer layers grown on silicon substrates. [2] The single crystalline quality of the films was particularly evident in the magnetization hysteresis loops, showing a sharp reversal at fairly high fields (120 Oe), when the samples were magnetized along the crystallographic easy axis. One technological disadvantage in this type of samples is the chemical interaction between the metallic layers and the silicon substrate.

In order to explore the possibility of epitaxial magnetic films on less reactive substrates, we studied the growth on $\mathrm{MgO}$ substrates. We have shown that it is possible to obtain epitaxial (001) and (111) Ni films grown on $\mathrm{MgO}$ substrates. [3] In particular we observed that the crystalline quality of the films improved considerably after $10 \mathrm{~nm}$ of film growth. We will now present our studies on the magnetic properties of these films, particularly the azimuthal dependence of the magnetization reversal using MOKRE, correlating our finding with the structural characterization obtained with RHEED, STM and XRD.
\end{abstract}

\section{INTRODUCTION}

In general, the magnetic properties, particularly the anisotropy, of epitaxial thin films are dominated by the crystallographic structure of the metal/substrate interface as well as the surface quality. In addition, for spin-dependent tunneling devices, the roughness at the surface must be very small in order to ensure the integrity of the subsequent deposition of ultra-thin, pinhole-free insulating layers. Thus, we have considered the growth of magnetic films on $\mathrm{MgO}$ substrates, which can be prepared with very smooth surfaces. [3]

Here we report on the magnetic switching behavior of these thin films. Our studies reveal the existence of a second-order transition from a single-domain state to a symmetry-broken multidomain state, which leads to singularities in the switching field along specific in-plane crystallographic directions, similarly to what we have observed for epitaxial $\mathrm{Co} / \mathrm{Cu}$ multilayers and $\mathrm{Co}$ thin films grown on silicon.

In our previous studies on $\mathrm{Co} / \mathrm{Cu}$ multilayers when considering the azimuthal dependence of the coercive field, we observed the presence of singular cusps along the crystallographic hard axes, with large coercive fields values, similar to the ones observed along the easy axes. We also observed fourfold symmetry for the (001) oriented samples and uniaxial symmetry for the (111) oriented ones. In order to understand these results we developed a phenomenological model starting with the traditional coherent rotation approach. [4] Details of this model are discussed elsewhere. [5] For clarity we will point out just the main features of the model. We start by considering the free energy density. For example, for $f c c$ structures in the (001) crystallographic orientation we may write:

$$
\mathrm{E}\left(\theta_{\mathrm{M}}, \mathrm{H}\right)=\mathrm{K}_{1} \cos ^{2}\left(\theta_{\mathrm{M}}\right) \sin ^{2}\left(\theta_{\mathrm{M}}\right)-\mathrm{H} \mathrm{M} \mathrm{M}_{\mathrm{s}} \cos \left(\theta_{\mathrm{M}}-\theta_{\mathrm{H}}\right)
$$


where $K_{1}$ represents the magnetocrystalline anisotropy [6] $M_{s}$ the saturation magnetization, $H$ the external applied field, and $\theta_{\mathrm{M}}\left(\theta_{\mathrm{H}}\right)$ are the angles between the Co [001] axis and the magnetization (external field), respectively. Stable orientations of the magnetization are found by minimizing $\mathrm{E}\left(\theta_{\mathrm{M}}, \mathrm{H}\right)$. To accurately describe the free energy for each sample, the anisotropy constants were determined from separate experimental measurements using ferromagnetic resonance (FMR). [7]

For the field applied along the easy axis, the simple coherent rotation model predicts switching of the magnetization from $\langle 110\rangle$ to $\left\langle 110\right.$ ) $>$ at $\mathrm{H}_{\mathrm{c}}={ }^{ \pm} 2 \mathrm{~K}_{1} / \mathrm{M}_{\mathrm{s}}$; however, the observed coercive field is much smaller. Thus we allow the system to form domains such that the moment reversal can occur at lower field by domain-wall nucleation. In this new scenario, we assume that the magnetization may "jump"[8] between two local free-energy minima when the gain in energy exceeds the cost of forming a domain wall:

$$
|\Delta \mathrm{E}| \geq \mathrm{E}_{\mathrm{w}}
$$

$E_{w}$ can be calculated straightforwardly using a standard form [9], which includes the usual exchange and anisotropy contributions. Using experimentally derived values for $\mathrm{K}_{1} / \mathrm{M}_{\mathrm{s}}$ (from FMR data), and assuming the bulk value for $\mathrm{M}_{\mathrm{s}}$, we were able to predict the coercive field and found it in close agreement with our experimental values.

For the (111)-oriented samples we note an interesting subtlety: the 3-fold magnetocrystalline anisotropy contribution cancels out so that any observed uniaxial symmetry is due to residual inplane switching due for example to a weak strain-induced uniaxial contribution.

To explain the singularities in coercive field observed for the external field applied along a hard axis direction we notice that what was initially a potential local minimum in energy density (for example for $\theta_{M}=0^{\circ}$ ) at large $\mathrm{H}$, transforms to a maximum as $\mathrm{H}$ is decreased, and two degenerate minima start to develop in close proximity and equidistant from each side of this new maximum. A similar situation occurs for $\theta_{M}=180^{\circ}$. Therefore, we see that as the external field is decreased from saturation, the system is no longer stable in the single-domain state, but a continuous transition to a multiple domain state occurs before the magnetization reversal is completed. The system spontaneously breaks up randomly in two types of symmetric domains, with the magnetization in each domain oriented along the direction of one of the two minima at each side of the hard axis direction, akin to a second-order phase transition. As the external field varies from $\mathrm{H}_{\mathrm{sat}}$ to $-\mathrm{H}_{\mathrm{sat}}$, our model predicts that the magnetization in each domain type will at first coherently rotate. When the jumping criterion (2) is satisfied (i.e. when the energy gain exceeds the cost of forming a domain wall for each domain) the magnetization jumps to the opposite set of minima, through nucleation of domain-walls. Since twice as many walls must now be nucleated, the energy requirement is higher than it would have been without the symmetry breaking, hence the 'spikes' in the coercive field.

We have obtained direct evidence for such a domain nucleation mechanism by performing MOKE microscopy during the magnetization reversal. The data show the sudden appearance of a broad distribution of stripe domains during the reversal, consistent with our domain-wall nucleation approach. Figure 1 shows the domain configuration during the magnetic switching when the field is applied along the easy and hard directions. We notice the smooth appearance of the domain walls in the easy direction [Fig. 1(a)], consistent with one single type of domain wall. On the other hand, along the hard axis [Fig.1 (b)], the domain-walls have a zig-zag appearance, 
as the magnetization must choose between the two equivalent symmetric orientations about the hard axis predicted by the model.
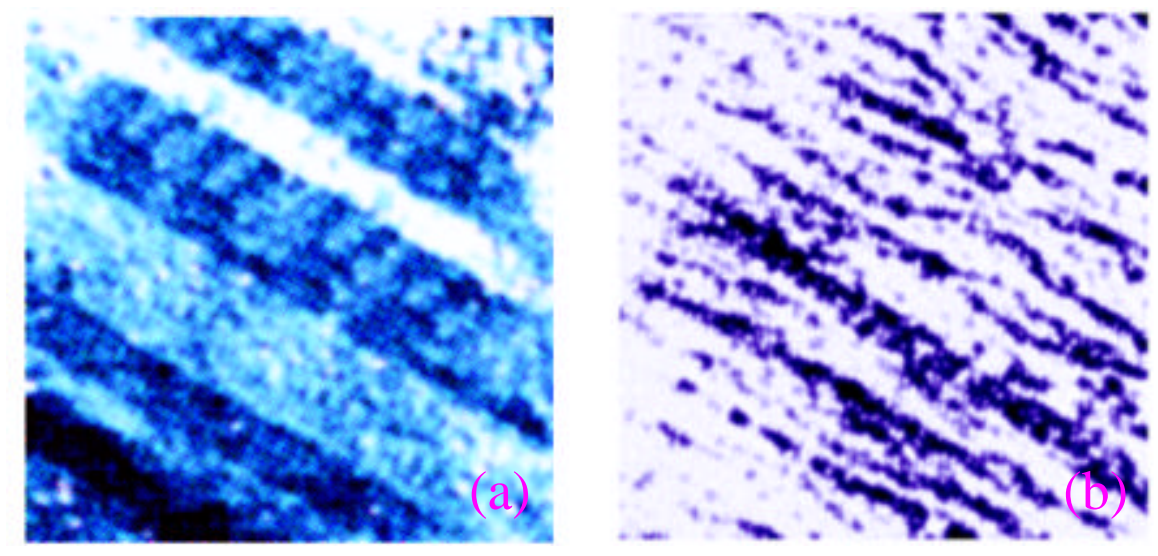

Figure 1. MOKE microscopy of a Co/Cu multiplayer. (a) Reversal of the magnetization along the easy axis, (b) reversal along the hard axis.

Thus, we have been able to qualitatively and quantitatively describe the magnetic moment switching for single crystalline $\mathrm{Co} / \mathrm{Cu}$ mulatilayers and $\mathrm{Co}$ thin films. Our new experimental data on the magnetization reversal of epitaxial single crystal Ni films further confirms this model as will be shown below.

\section{EXPERIMENTAL DETAILS}

The Ni films were grown in an MBE VG $80 \mathrm{M}$ system with a background pressure $<5 \times 10^{-11}$ torr. Ni was evaporated from a $99.999 \%$ pure source. The deposition rate was $0.5 \AA / \mathrm{sec}$. The substrates used in the experiment were $0.5 \mathrm{~mm}$ thick, $1 \times 1 \mathrm{~cm}^{2}$ pre-polished $\mathrm{MgO}(001)$ and (111) oriented single crystals, which were heat-treated in UHV at $800^{\circ} \mathrm{C}$ for $1 \mathrm{hr}$. The combination of flat polished substrates and the UHV heating cycle to allow the surface layers to regain crystalline order has been proven to permit growth of single crystal metal films [6] as well as exhibiting sharp reflection high-energy electron diffraction (RHEED) from the MgO surface.

$E x$-situ atomic force microscopy (AFM) characterization of the annealed surfaces showed smooth surfaces with a root mean square (rms) surface roughness of $0.2 \mathrm{~nm}$ for the (001) oriented substrates and $0.5 \mathrm{~nm}$ for the (111) oriented ones. Prior to initiating the growth, the substrate temperature was lowered to the appropriate deposition temperature for metal growth $\left[\mathrm{T} \cong 100{ }^{\circ} \mathrm{C}\right.$ for $(001)$ and $\mathrm{T} \cong 300{ }^{\circ} \mathrm{C}$ for (111) oriented Ni films]. Heat transfer was by direct radiation between the heater and $\mathrm{MgO}$ substrate. The RHEED patterns were recorded continuously during deposition and during subsequent annealing of the films [9]. The surface morphology of the asdeposited and annealed films was determined in-situ with scanning tunneling microscopy [(STM) RHK model STM100]. The structural characterization of the films is extensively discussed elsewhere. [3] 


\section{DISCUSSION}

Let us just review the STM studies on the (001) surface, which indicated "stripe" reconstruction, because of the possible repercussion on the magnetic properties. In order to understand this reconstruction we considered the possible effect of strain. The lattice misfit between $\mathrm{MgO}$ and $\mathrm{Ni}$ is $16 \%$. However, it has been postulated [6] that an in-plane super-cell matching (commensuration) between the film and substrate with $\mathrm{a}_{\mathrm{o}}(\mathrm{Ni})$ x $6=2.0446 \mathrm{~nm}$ and $\mathrm{a}_{\mathrm{o}}(\mathrm{MgO}) \times 5=2.1066 \mathrm{~nm}$ will reduce the misfit to $\sim 0.8 \%$. The critical thickness needed to relieve such a small strain may be quite large. Still, some authors have claimed that super-cell matching itself cannot give rise to the formation of single crystalline Ni layers, as it has been shown that in other cases interfacial periodic reconstructions can exist that allow for single crystal growth. Our observations support this. Annealing the films relaxed the surface and evidenced a reconstruction with periodicity related to the size of the postulated super-cell (i.e. $2.1 \mathrm{~nm}$ ). [Figure 2] This type of surface reconstruction may have also an effect on the magnetic anisotropy, in the form of a weak uniaxial contribution, as will be shown below. No reconstruction was observed on the (111) oriented films after annealing.

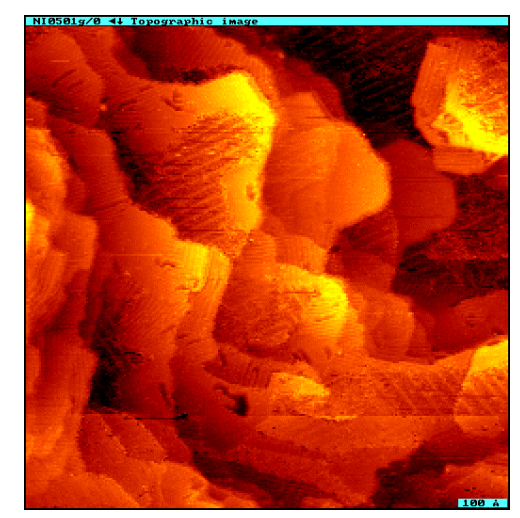

Figure2. STM image of a (001) Ni film after annealing. The bar corresponds to $10 \mathrm{~nm}$.

The magnetization reversal of these films was studies with MOKE. Our setup allows the rotation of the sample in the plane of the applied field in order to facilitate studies on the azimuthal dependence of the reversal. Figure 3 shows the azimuthal dependence of the coercive field for (001) Ni films (40 nm thick) grown on $\mathrm{MgO}$ and further annealed. We observe the singular "spikes" along the hard axes, the four-fold symmetry due to the magnetocrystalline anisotropy, and also a weak uniaxial contribution possibly due to the "stripe" reconstruction mentioned above.

The azimuthal dependence of the (111) films didn't show any singularities, indicating that possible uniaxial contributions due to strain, steps, etc, are absent. This is consistent with the lack of observation of any type of reconstruction on the (111) surface and also with the smooth quality of the surface. The thickness of the films (in the 30-50 $\mathrm{nm}$ range) precludes the presence of strain, contrary to what we observed in our previous study on (111) $\mathrm{Co} / \mathrm{Cu}$ multilayers. We did observe a weak three-fold anisotropy, due to the magnetocrystalline anisotropy, which was evident probably due to a slight misalignment between the plane of the sample and the applied field (Figure 4). Thus, the present data corroborates our magnetization switching model. 


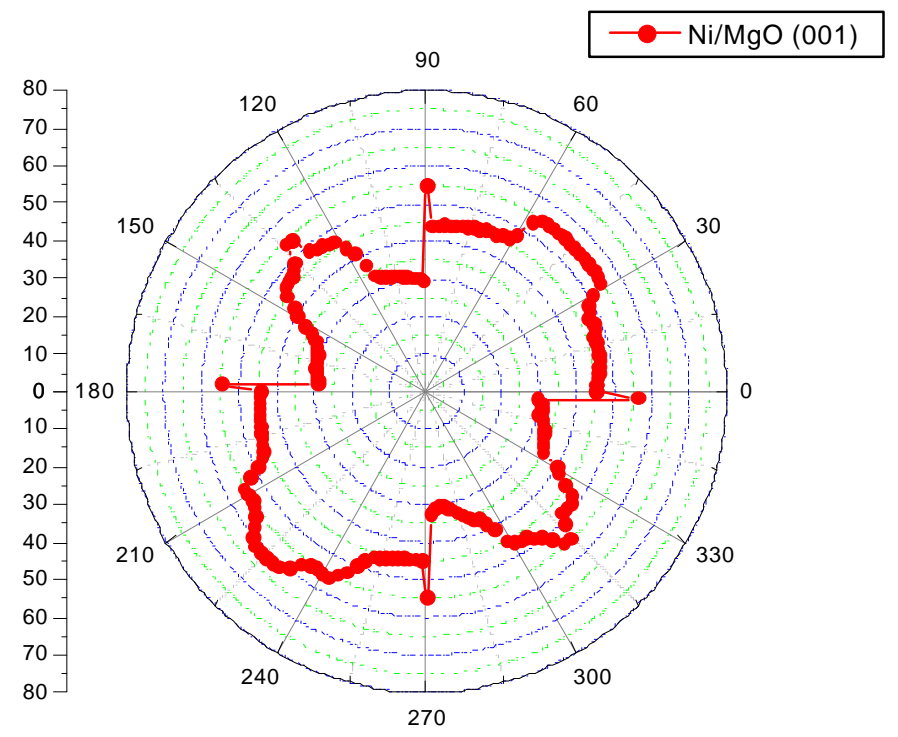

Figure 3. Azimuthal dependence of the coercive field for a (001) Ni film. The vertical axis indicates the coercive field $(\mathrm{Oe})$.

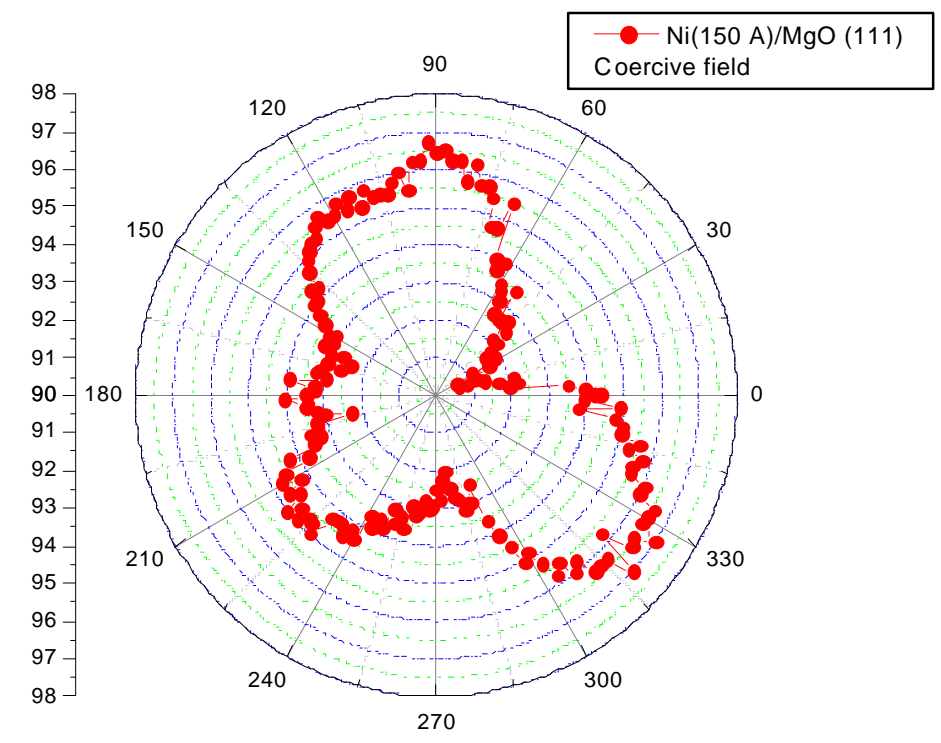

Figure 4. Azimuthal dependence of the coercive field for a (111) Ni film. The vertical axis indicates the coercive field $(\mathrm{Oe})$. 


\section{References and Notes}

[1]. R. A. Lukaszew, Y. Sheng, C. Uher and R. Clarke, Appl. Phys. Lett. 75, 1941 (1999).

[2]. R. A. Lukaszew, Y. Sheng, C. Uher and R. Clarke, Appl. Phys. Lett. 76, 724 (2000).

[3]. R. A. Lukaszew, V. Stoica, C. Uher and R. Clarke, MRS Proceedings, Fall 2000.

[4]. E. C. Stoner and E. P. Wohlfarth, Phil. Trans. Roy. Soc. A-240, 599-644 (1948). [two authors]

[5]. R. A. Lukaszew, E. Smith, R. Naik and R. Clarke, submitted to Phys. Rev. Lett. (2001)

[6]. $\mathrm{K}_{1}$ is negative for $f c c$ Co making the in-plane [001] direction a magnetic hard axis.

[7]. R. A. Lukaszew, R. Naik, K. R. Mountfield and K. R. Artman, J. Appl. Phys. 79, 47874789 (1996). [four authors]

[8]. J. M. Florczak and E. D. Dahlberg, Phys. Rev. B 44, 9338-9347 (1991). [two authors]

[9]. R. F. Soohoo, Magnetic Thin Films, (Harper and Row, New York, 1965); M. Prutton,

Thin Ferromagnetic Films, (Butterworths, Washington, 1964). 